Meta

Journal des traducteurs

Translators' Journal

\title{
Le moteur Wankel : principe et terminologie
}

\section{Jacques Lethuillier}

Volume 26, numéro 4, décembre 1981

URI : https://id.erudit.org/iderudit/003484ar

DOI : https://doi.org/10.7202/003484ar

Aller au sommaire du numéro

Éditeur(s)

Les Presses de l'Université de Montréal

ISSN

0026-0452 (imprimé)

1492-1421 (numérique)

Découvrir la revue

Citer cet article

Lethuillier, J. (1981). Le moteur Wankel : principe et terminologie. Meta, 26(4), 369-376. https://doi.org/10.7202/003484ar d'utilisation que vous pouvez consulter en ligne.

https://apropos.erudit.org/fr/usagers/politique-dutilisation/ 


\section{LE MOTEUR WANKEL : PRINCIPE ET TERMINOLOGIE}

Les moteurs rotatifs (rotary engine, rotary combustion engine, r.c. engine) sont des moteurs à combustion interne (internal combustion engine, i.c. engine) comme le classique moteur à pistons (piston engine, reciprocating engine). Il s'agit donc de machines thermiques (heat engine) motrices où l'air non seulement sert de fluide moteur (working fluid, working medium, working substance), mais encore participe de façon directe à la phase dite d'apport de chaleur (heat addition, heat input) en jouant le rôle de comburant (comburent, oxidizer) dans un processus de combustion.

Ces moteurs possèdent une autre caractéristique les rapprochant du moteur à pistons : ils font partie de la même grande famille des moteurs volumétriques ou moteurs à capsulisme (displacement-type engine), qui utilisent la détente (expansion) d'un gaz dans une enceinte fermée dont le volume est variable pour produire de l'énergie motrice.

Les quatre phases caractéristiques du cycle d'évolution du fluide moteur dans une machine thermique, à savoir la compression, l'apport de chaleur, la détente et la restitution de la chaleur résiduelle (heat rejection) à la source froide (cold body), se déroulent à l'intérieur d'un même organe polyvalent (ici, l'ensemble piston rotatif et carter-enveloppe), selon un processus séquentiel.

En ce sens, on peut les opposer aux moteurs cinétiques ou turbomachines (non-displacement type engine) comme la turbine à gaz (gas turbine engine), qui comportent autant d'organes spécialisés, en communication les uns avec les autres, que de phases à mettre en œuvre et qui sont le siège d'un processus continu - toutes les phases se déroulant simultanément, sans interruption, dans des organes distincts, présentant la caractéristique d'être ouverts.

En quoi se distinguent alors les moteurs rotatifs et, plus particulièrement, le moteur Wankel (Wankel engine, eccentric-rotor engine), du classique moteur à pistons? Le caractère distinctif de ces machines, c'est le fait qu'elles permettent d'obtenir directement la rotation continue d'un arbre moteur (crankshaft), et non par conversion du mouvement rectiligne alternatif (reciprocating motion) d'un piston crosse ou piston fourreau (trunk piston), faisant office d'organe mécanique récepteur, au moyen d'un système bielle-manivelle (rotating slider crank mechanism), encore appelé attelage moteur (piston and rod assembly). Il s'en- 
suit que disparaît le traditionnel équipage mobile (moving parts) dont l'équilibrage (balancing) est si difficile à réaliser en raison des mouvements complexes pris par les pièces de liaison qu'il met en jeu.

L'ensemble bielle-manivelle (piston and rod assembly), dans le moteur Wankel, est remplacé par un piston rotatif ou rotor (rotor), qui est un disque affectant la forme d'un triangle équilatéral, mais dont les côtés sont légèrement convexes. Il tourne à l'intérieur d'une chambre trochoïdale, le carter-enveloppe (rotor housing, epitrochoidal chamber), limitée par deux flasques (end plate, side housing). Les faces périphériques du rotor, du fait que les sommets ou pointes (apex, tip) restent constamment en contact avec la paroi interne du carterenveloppe, y définissent trois chambres de travail ou chambre de combustion à volume variable réalisant un capsulisme.

L'admission et l'échappement se font par des lumières (port) toujours ouvertes, ménagées d'un côté du carter, à la hauteur de l'étranglement. Le passage d'un sommet du rotor devant une lumière marque le début de la mise en communication avec le carburateur ou l'atmosphère pour une chambre, et la fin de la communication pour la chambre qui précède. Dans la zone du carter diamétralement opposée aux lumières - où le volume des chambres définies par chacune des faces du rotor devient le plus restreint et où se produit donc la combustion -, sont disposées une ou deux bougies d'allumage (spark plug, ignition plug).

Une cavité (recess) est ménagée sur chaque face périphérique du rotor. De sa capacité dépend l'espace mort ou volume nuisible (dead space, clearance space) et, par conséquent, le rapport volumétrique de compression (compression ratio) du moteur.

L'étanchéité des chambres de travail est obtenue grâce à des joints : segments de flanc ou segments périphériques (side seal) empêchant les fuites latérales; segments d'arête ou segments radiaux (apex seal) isolant les chambres les unes des autres; et segments d'angle (corner seal) à la réunion des deux. Des barillets (retainer pin) à chaque sommet et de chaque côté servent à maintenir entre eux des groupes de trois segments.

Le moteur Wankel fonctionne selon un cycle à quatre temps. Pour un tour du rotor sur lui-même, il se produit trois cycles complets : un par chambre. Et l'arbre moteur pendant ce temps effectue trois révolutions.

On peut suivre la séquence d'événements se déroulant à l'intérieur de la chambre $\mathrm{BA}$ à partir du moment où le segment d'arête de tête $\mathrm{A}$ découvre la lumière d'admission (intake port). Par suite de la rotation du rotor, le volume de cette chambre augmente. Il se crée une dépression (vacuum) qui provoque l'aspiration du mélange carburé. L'admission se poursuit jusqu'au passage du segment d'arête B devant la lumière d'admission. Commence alors la compression, qui se termine quand la chambre occupe un espace minimal. À ce moment, la face $\mathrm{AB}$ étant presque parallèle à la paroi du carter-enveloppe, des étincelles jaillissent entre les électrodes des bougies. C'est le début du temps moteur, durant lequel les gaz de la combustion exercent une poussée sur la face du rotor délimitant la chambre. La fin de la détente des gaz coïncide avec le passage de l'arête A devant la lumière d'échappement (exhaust port), qui marque aussi le début de la 
phase d'échappement. Puis l'arête A repassera par son point de départ, la lumière d'admission, et un nouveau cycle recommencera.

Le piston rotatif comporte un évidement circulaire et concentrique grâce auquel la poussée reçue par ses faces périphériques se trouve transmise à l'arbre moteur. Ce dernier est supporté par deux paliers disposés au centre géométrique des flasques fermant le carter de chaque côté. Entre les deux paliers il comporte un excentrique (eccentric, bulge) en contact permanent avec l'évidement central du rotor qui, d'une certaine façon, joue le rôle du maneton (crankpin) d'un vilebrequin classique.

Par ailleurs, un engrenage réalise l'asservissement du mouvement de rotation sans glissement du rotor à celui de l'excentrique. Il se compose d'une roue à denture intérieure montée au centre du rotor et en prise constante avec un pignon fixe plus petit, solidaire de l'un des flasques.

Le moteur Wankel offre l'avantage de posséder une bonne puissance massique (power-to-weight ratio) et un encombrement réduit. On parvient à l'équilibrer parfaitement et donc à éliminer presque toutes les vibrations, qui représentent une cause d'usure très importante dans les classiques machines à pistons. Pourtant, malgré ces qualités et en dépit du fait que plusieurs constructeurs en aient équipé certains de leurs modèles pendant plusieurs années, la solution du rotatif ne s'est pas imposée pour la traction routière. Sans doute faut-il expliquer ce demi-succès par la faiblesse du rendement obtenu pour ce type de moteur jusqu'à présent.

\section{LEXIQUE ANGLAIS-FRANÇAIS}

Apex - sommet, pointe. Chacune des arêtes à l'intersection de deux faces périphériques du rotor. Apex seal - segment d'arête, segment radial. Joint métallique disposé à un sommet du rotor et s'appliquant contre la paroi de la chambre trochoïdale ou carter-enveloppe. Il isole une chambre de travail de la suivante.

Balancing - équilibrage. Fixation de contrepoids sur un arbre, de façon que le centre de gravité de l'équipage mobile soit sur l'axe de rotation. On cherche ainsi à compenser les forces d'inertie alternatives des attelages moteurs.

Bulge - Voir eccentric.

Clearance space - espace mort, espace résiduel, volume nuisible. Espace minimal d'une chambre de combustion dans un moteur de type volumétrique.

Cold body - source froide. Milieu auquel un moteur, après avoir transformé en travail une partie de la chaleur reçue d'une source chaude, restitue la chaleur résiduelle qui ne peut être convertie en énergie mécanique d'après le théorème de Carnot.

$$
\begin{aligned}
& \text { source chaude } \\
& \text { apport de chaleur } \\
& \text { MOTEUR travail } \\
& \text { chaleur restituée }
\end{aligned}
$$

source froide

Comburent - comburant. Substance fournissant l'oxygène nécessaire à la combustion d'un corps combustible. Il s'agit de l'air de l'atmosphère dans le cas des moteurs utilisés pour la traction routière. 
Compression ratio - rapport volumétrique de compression, taux de compression. Pour un moteur à combustion interne de type volumétrique, rapport entre le volume de la charge en début de compression et son volume en fin de compression.

Compression space - Voir clearance space.

Corner seal - segment d'angle. Chacun des joints d'étanchéité glissant sur un flasque, aux extrémités d'un segment d'arête.

Crankpin - maneton. Partie excentrée d'un vilebrequin de moteur à pistons, sur laquelle s'articule la tête de bielle.

Crankshaft - arbre moteur, vilebrequin, arbre-excentrique.

Dead space - Voir clearance space.

Displacement-type engine - moteur volumétrique, moteur à capsulisme. Moteur réalisant la conversion de l'énergie potentielle d'un fluide par détente de celui-ci dans une enceinte fermée dont le volume est variable.

Eccentric - excentrique. Disque excentré, solidaire de l'arbre moteur, qui joue le rôle du maneton dans un moteur à pistons classique. Il transmet le mouvement du piston rotatif à l'arbre.

Eccentric-rotor engine - moteur à piston rotatif Wankel, moteur Wankel. Moteur à combustion interne de type volumétrique, dont le capsulisme est réalisé par un piston rotatif (ayant la forme d'un disque triangulaire) qui tourne à l'intérieur d'un carter trochoïdal.

Eccentricity - excentricité, excentration. Dans un moteur Wankel, distance entre le centre de l'excentrique et l'axe de rotation de l'arbre moteur.

End plate - flasque, carter latéral. Chacune des plaques fermant latéralement le carter-enveloppe et servant en outre de palier pour l'arbre-excentrique.

Epitrochoidal chamber - chambre trochoidale, carter-enveloppe, stator. Dans un moteur Wankel, pièce fixe ayant la forme d'un cylindre à deux lobes, où tourne le piston rotatif de forme triangulaire que traverse l'arbre-excentrique.

Expansion - détente. Augmentation du volume occupé par un fluide gazeux et diminution de sa pression. Au cours du phénomène, l'énergie de pression se transforme en énergie cinétique ou énergie de vitesse. Dans les moteurs thermiques, la détente d'un fluide moteur, auquel on a préalablement apporté de la chaleur, est utilisée pour produire du travail. L'énergie cinétique acquise par le fluide en se détendant est cédée à un organe mécanique récepteur, roue de turbine ou piston, à l'intérieur d'une enceinte ouverte ou fermée. C'est la troisième phase du cycle d'évolution décrit par le fluide moteur, phase de conversion proprement dite de la chaleur en travail, ou conversion thermodynamique.

Exhaust port - Iumière d'échappement. Voir Port.

Gas turbine engine - turbine à gaz. Moteur à combustion interne de type non volumétrique, c'est-àdire s'apparentant aux turbomachines. Les quatre phases du cycle décrit par le fluide moteur (l'air dans ce cas) s'y déroulent en continu, à l'intérieur d'organes communiquant entre eux et formant un circuit ouvert.

Heat addition - apport de chaleur. Deuxième phase du cycle d'évolution d'un fluide moteur dont une machine thermique est le siège. Elle peut avoir lieu à l'extérieur ou non de la machine où le travail est véritablement produit. Selon le cas, il s'agit d'un moteur à combustion interne ou externe.

Heat engine - moteur thermique. Machine produisant du travail (énergie motrice) à partir de chaleur. La conversion se fait par le biais d'un fluide moteur qui reçoit de l'énergie calorifique d'une source chaude ou directement en participant à une combustion. Une partie seulement de la chaleur reçue est transformée en travail quand le fluide moteur se détend dans une capacité contenant un organe mécanique récepteur (piston ou roue de turbine). La chaleur résiduelle est restituée à une source froide, que peut représenter l'environnement, c'est-à-dire l'atmosphère.

Heat input - Voir heat addition.

Heat rejection - restitution de la chaleur résiduelle. Quatrième phase du cycle d'évolution du fluide moteur dans une machine thermique.

Hot body - source chaude. Milieu auquel le fluide moteur mis en cuvre dans une machine thermique motrice emprunte des calories que celle-ci transforme ensuite partiellement en travail.

Ignition plug - bougie d'allumage. Dans un moteur à combustion interne de type volumétrique, dispositif électrique faisant partie d'un circuit d'allumage externe et conçu pour produire à intervalles réguliers une étincelle de façon que s'établisse un régime de combustion intermittent.

Intake port - lumière d'admission. Voir Port.

Internal combustion engine - moteur à combustion interne. Machine thermique motrice où l'air non seulement joue le rôle de fluide moteur, mais encore participe de façon directe à la phase dite d'ap- 
port de chaleur en jouant le rôle de comburant dans une combustion. On retrouve les quatre phases caractéristiques du cycle d'évolution du fluide moteur au sein d'une machine thermique, à savoir la compression, l'apport de chaleur, la détente et la restitution de la chaleur résiduelle à une source froide. Ce qui est particulier, c'est le fait que la chaleur - que ce type de machine a pour fonction de transformer en travail - est dégagée au sein du fluide moteur lui-même, par combustion.

I.c. engine - Voir internal combustion engine.

Moving parts - équipage mobile. Dans un moteur à pistons classique, ensemble formé par l'arbre équipé de ses bielles et pistons.

Non-displacement type engine - turbomachine réceptrice, turbomoteur, machine cinétique. Machine prélevant de l'énergie à un flux continu de fluide, qu'elle transforme en énergie mécanique utilisée pour maintenir en mouvement une autre machine.

Oxidizer - Voir comburent.

Piston and rod assembly - attelage moteur, ensemble piston-bielle.

Piston engine - moteur à pistons. Moteur à combustion interne où l'organe primaire recevant l'énergie du fluide moteur, quand il se détend dans une enceinte fermée à volume variable, est constitué par un piston de forme cylindrique qui décrit un mouvement rectiligne alternatif.

Port - lumière. Chacun des deux orifices, non munis d'un dispositif obturateur, qui mettent successivement en communication avec l'atmosphère les trois chambres de travail réalisées entre le rotor et le stator d'un moteur Wankel, par l'intermédiaire du carburateur pour la lumière d'admission et par l'intermédiaire de l'échappement pour la lumière d'échappement.

Power-to-weight ratio - puissance massique. Grandeur caractéristique d'un moteur, traduisant la puissance qu'il développe par unité de masse. On l'utilise pour établir des comparaisons entre les différents types de moteurs, au chapitre de la pénalité introduite par le poids.

R.c. engine - Voir rotary combustion engine.

Recess - alvéole, cavité. Évidement ménagé à la périphérie du rotor d'un moteur Wankel, sur chacune de ses faces. Son importance conditionne le rapport volumétrique de compression.

Reciprocating engine - Voir Piston engine.

Reciprocating motion - mouvement rectiligne alternatif. Mouvement de va-et-vient dont s'anime le piston dans un moteur du type à pistons.

Retainer pin - barillet. Petite pièce de forme cylindrique sur laquelle s'ajustent les extrémités des segments de flanc (assurant l'étanchéité des chambres de travail dans un moteur Wankel). Elle assure la jonction délicate entre les segments d'angle et les segments de flanc.

Rotor - piston rotatif, rotor. Disque affectant la forme d'un triangle équilatéral, mais dont les arêtes sont légèrement bombées. Ses trois faces, qui délimitent chacune une chambre de travail à volume variable dans le carter-enveloppe ou stator, reçoivent successivement la poussée accompagnant la détente des gaz de combustion, qu'elles transmettent à l'arbre moteur par l'intermédiaire d'un excentrique de ce dernier.

Rotor housing - Voir epitrochoidal chamber.

Rotary combustion engine - moteur rotatif. Moteur à combustion interne de type volumétrique, où l'organe moteur primaire est animé d'un mouvement de rotation et non d'un mouvement alternatif comme dans les machines à pistons classiques. De tous les moteurs rotatifs, le plus courant est désormais le moteur Wankel.

Rotary engine - Voir rotary combustion engine.

Rotating slider crank mechanism - système bielle-manivelle, mécanisme bielle-manivelle. Dans un moteur à pistons, mécanisme articulé servant à convertir en un mouvement rotatif du vilebrequin le mouvement rectiligne alternatif des pistons. Il est formé de deux bras - l'axe de piston qui, avec le piston, fait office de coulisseau, et la manivelle calée sur le vilebrequin - reliés entre eux par une bielle.

Side housing - Voir end plate.

Side plate - Voir end plate.

Side seal - segment de flanc, segment périphérique. Chacun des joints métalliques disposés sur les bords des trois faces du rotor (côtés du triangle curviligne) et destinés à assurer l'étanchéité entre les chambres de travail et les carters latéraux.

Single-rotor Wankel engine - moteur Wankel monorotor. Moteur Wankel comportant un rotor unique et, par conséquent, une seule chambre trochoïdale.

Spark plug - Voir ignition plug.

Tip - Voir apex. 


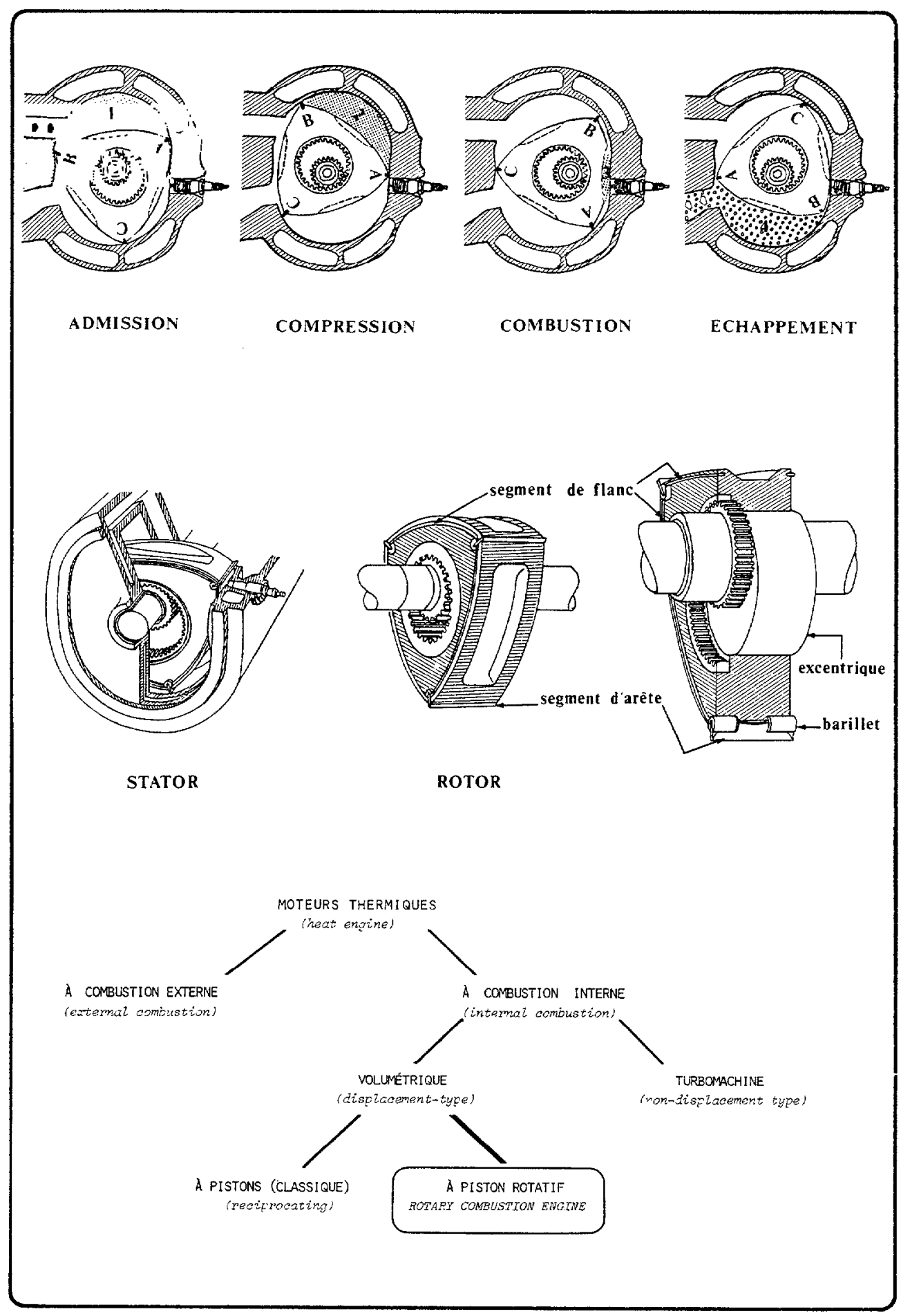


Trunk piston - piston crosse, piston fourreau. Piston creux et ouvert d'un côté, auquel le pied de bielle s'articule directement par le biais de l'axe de piston.

Two-rotor Wankel engine - moteur Wankel birotor. Moteur Wankel comportant deux rotors ou pistons rotatifs, disposés dans des chambres trochoïdales contiguës mais séparées par un flasque médian.

Vacuum - dépression.

Wankel engine - Voir eccentric-rotor engine.

Working agent - fluide moteur, agent moteur. Dans un moteur thermique, fluide recevant de l'énergie sous forme de chaleur d'une source chaude et la restituant en partie sous forme de travail à un organe mécanique récepteur (piston ou roue de turbine) et en partie sous forme d'énergie thermique résiduelle à une source froide.

\section{LEXIQUE FRANÇAIS-ANGLAIS}

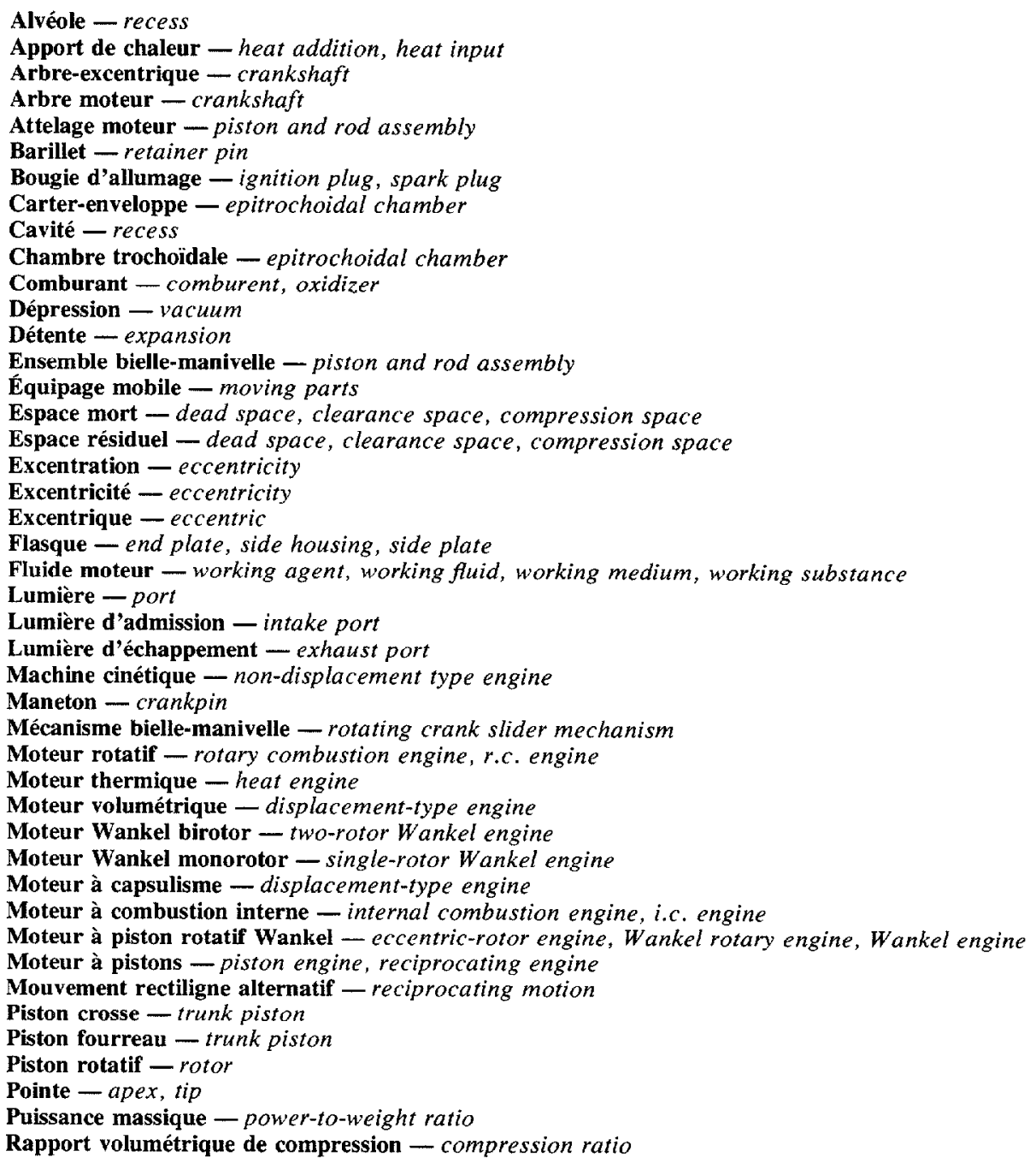


Restitution de la chaleur résiduelle - heat rejection

Rotor - rotor

Segment d'angle - corner seal

Segment d'arête - apex seal

Segment de flanc - side seal

Segment périphérique - side seal

Segment radial - apex seal

Sommet - apex, tip

Source chaude - hot body

Source froide - cold body

Stator - rotor housing

Système bielle-manivelle. - rotating slider crank mechanism

Taux de compression - compression ratio

Turbine à gaz - gas turbine engine

Turbomachine réceptrice - non-displacement type engine

Vilebrequin - crankshaft

Volume nuisible - compression space, dead space, clearance space 